\section{CRC: zuverlässiges Screening auf MMR-Defizienz}

\author{
Bei den meisten Patienten mit kolorektalen Karzinomen (CRC) wird \\ empfohlen, ein Lynch-Syndrom-Screening durchzuführen. Bei meta- \\ stasiertem CRC müssen für die Wahl der richtigen Therapie zudem der \\ RAS- und BRAF-Status bestimmt werden. Mithilfe des "next generation \\ sequencing" (NGS) kann dies in einem Schritt erfolgen.
}

$\mathrm{B}^{\mathrm{c}}$ ei rund $15-20 \%$ der kolorektalen Karzinome weisen die Tumoren einen Mangel an DNA-Mismatch-Reparaturproteinen (MMR-D) auf. Bei einem Viertel der Betroffenen geht die MMR-D auf das Lynch-Syndrom zurück, ein autosomal dominant vererbtes Krankheitsbild mit Keimbahnmutationen derjenigen Gene, die für MMR-Proteine kodieren. In Leitlinien wird heute daher ein Screening auf Lynch-Syndrom entweder anhand der MMR-Proteinexpression oder der Mikrosatelliteninstabilität (MSI) gefordert. Eine MMR-D, die auch sporadisch auftreten kann, ist einerseits mit einer besseren Prognose verbunden und bestimmt andererseits auch das Ansprechen auf die Therapie. Die Patienten sprechen nicht auf 5-Fluorouracil an, wohl aber auf Immuncheckpointinhibitoren.

Zsofia K. Stadler und Kollegen bestimmten bei 224 CRC-Patienten am Memorial Sloan Kettering Cancer Center, New York, die NGS-Mutationsprofile und die Mutationslast und verglichen die Resultate mit dem MMR-Status, wie er sich aus der Routine-Immunhistochemie (IHC) ergab.

Von den 224 CRC-Patienten zeigten $13 \%(\mathrm{n}=28)$ in der IHC eine MMR-Defizienz. Mit NGS (341 Gene) waren $100 \%$ von 193 Tumoren mit $<20$ Mutationen MMR-profizient (MMR-P). Von $31 \mathrm{Tu}$ moren mit $\geq 20$ Mutationen erwiesen sich 28 (90\%) als MMR-defizient (MMR-D).

Die 3 übrigen waren MMR-P und hoben sich von der MMR-D-Gruppe mit jeweils $>150$ Mutationen deutlich ab. Jeder dieser 3 Tumoren beherbergte eine P286R-hotspot-POLE-Mutation, gleichbedeutend mit dem Ultramutator-Phänotyp. Die MMR-D-Karzinome hatten median 50 Mutationen, verglichen mit 6 bei den MMR-P-Tumoren mit POLEWildtyp $(\mathrm{p}<0,001)$.

Mit Cutoff-Werten der Mutationslast von $\geq 20$ und $<150$ für die MMR-D-Detektion betrugen sowohl Sensitivität als auch Spezifität 1,0.

Fazit: NGS wird heute vielfach zur Tumor-Genotypisierung eingesetzt und liefert auch Daten zur Gesamtmutationslast des Tumors. Durch Festlegung von Grenzwerten für die Mutationslast, lässt sich mit demselben Testsystem auch ein sehr genaues Screening auf eine MMR-Defizienz durchführen. Diese spielt sowohl für die Prognose als auch das Therapieansprechen eine groBe Rolle.

Brigitte Schalhorn

Stadler ZK et al. Reliable detection of mismatch repair deficiency in colorectal cancers using mutational load in next-generation sequencing panels. J Clin Oncol. 2016;34(18):2141-7.

\title{
Peptidrezeptor-Radionuklid-Therapie bei neuroendokrinen Tumoren
}

Die Wirksamkeit einer Peptidrezeptor-Radionuklid-Therapie, die sich gegen den Somatostatin-Rezeptor von neuroendokriner Tumoren richtet, wurde bisher eher monozentrisch und retrospektiv untersucht. Nun gibt es erstmals Ergebnisse einer prospektiven, multizentrischen Register-Studie.

V on 450 Patienten mit progredienten, lokal fortgeschrittenen oder metastasierten, niedrig- oder mittelgradig differenzierten neuroendokrinen Tumoren (NET) mit Überexpression des Somatostatin-Rezeptors aus 5 deutschen Zentren konnten 439 ausgewertet werden. $73 \%$ der Patienten waren mit mindestens einer Therapie vorbehandelt. Die Primärtumoren lagen meist im Pankreas (38\%), Dünndarm (30\%) oder den Bronchien (4\%), bei $19 \%$ war die Lokalisation unbekannt.

Die Peptidrezeptor-Radionuklid-Therapie erfolgte gemäß den aktuellen Leitlinien. 54\% der Patienten wurden mit Lutetium-177 behandelt, $17 \%$ mit Yttri- um-90 und 29\% mit beiden Radionukliden.

Nach median 24,4 Monaten betrug das mediane Gesamtüberleben (OS) aller Patienten 59 Monate. Bei Patienten, die nur Yttrium-90 erhalten hatten, war das mediane OS signifikant niedriger als bei jenen, die mit Lutetium-177 allein oder in Kombination behandelt wurden (Hazard Ratio [HR] 3,22). Patienten mit NET-Grad II bzw. III hatten ein signifikant schlechteres OS als Patienten mit Grad-I-Tumoren (HR 2,06 bzw. 4,22). Außerdem lebten Patienten mit kleinen NET im Dünndarm signifikant länger (HR 0,39) als Patienten mit anderen Lokalisationen. Das mediane progressions- freie Überleben betrug 41 Monate insgesamt und war signifikant kürzer bei $\mathrm{Pa}$ tienten, die nur Yttrium erhalten hatten (HR 2,7). Eine vollständige Remission erreichten 5,6\% der Patienten, 22,4\% hatten eine partielle Remission und 47,3\% eine stabile Erkrankung. Bei 4\% der Patienten schritt die Erkrankung weiter fort. Unerwünschte Ereignisse am Knochenmark oder Beeinträchtigungen der Nierenfunktion vom Grad $\geq 3$ traten bei $0,2-1,5 \%$ der Patienten auf.

Fazit: Die Peptidrezeptor-RadionuklidTherapie ist für Patienten mit niedrig- bis mittelgradigen neuroendokrinen Tumoren eine hocheffektive Therapie mit geringen Nebenwirkungen. Judith Neumaier

Hörsch D et al. Effectiveness and side-effects of peptide receptor radionuclide therapy for neuroendocrine neoplasms in Germany: A multi-institutional registry study with prospective follow-up. Eur J Cancer. 2016;58:41-51. 